\title{
Dental Perspective on Biomedical Waste and Management - A Knowledge, Attitude, and Practice Survey: A Cross-sectional Study
}

\author{
K. Srinivasan \\ CKS Theja Institute of Dental Science and Research Centre, Tirupati, Andhra Pradesh, India
}

Email for correspondence: skskskpedo@gmail.com

\begin{abstract}
Background: The biomedical waste is known as the second dangerous waste in the world that needs to be properly managed. Since the implementation of the Biomedical Waste Management (BMW) Rules, 1998, every concerned health personnel is expected to have proper knowledge, practice, and capacity to guide others for waste collection and management, and proper handling techniques. Aims and Objectives: The present study was planned to evaluate the practical calibration and awareness of dental surgeons in disposal of hazardous biomedical waste generated during dental treatment into color-coded disposing bags at a dental clinic. Materials and Methods: A cross-sectional, a pre-tested, semi-structured questionnaire-based survey containing 53 questions to assess the knowledge, attitude, and practice on BMW among dental surgeons was planned. Results were expressed as a number and percentage of respondents for each question and Chi-square test was performed for inferential statistical analysis with $P<0.05$ indicating level of statistical significance. Results: Results showed that a large percentage of the dentist was not aware of the process of BMW (89\%), whereas about half of the subjects were moderate to slightly aware of the recycling/ reusing of dental materials. Conclusion: The study revealed that knowledge, attitude, and practice regarding BMW among were low. Periodical sensitization and training program should be conducted for health-care providers.
\end{abstract}

Key words: Attitude, biomedical waste, dental health-care personnel, knowledge, self-administered questionnaire, waste management

\section{INTRODUCTION}

The essences of cleanliness were captured by the Dravidians, who in 5000 B.C gave due emphasis to safe and effective sewerage systems, to get rid of all solid and liquid waste generated by the population. They were indeed the pioneers as far scientific waste management is considered. ${ }^{[1]}$

The World Health Organization (WHO) defines health-care waste as total waste generated by

\begin{tabular}{|c|c|}
\hline \multirow{3}{*}{ Quick Response Code } & Article Info: \\
\hline & doi: $10.5866 / 2019.11 .10001$ \\
\hline & $\begin{array}{l}\text { Received: 04-01-2019 } \\
\text { Revised: 07-02-2019 } \\
\text { Accepted: 03-03-2019 } \\
\text { Available Online: 01-04-2019, (www. } \\
\text { nacd.in)@ NAD, 2019 - All rights } \\
\text { reserved }\end{array}$ \\
\hline
\end{tabular}

hospitals, health-care establishments, and research facilities in the diagnosis, treatment, or immunization of human beings or animals, and other associated research and services. ${ }^{[2]}$

According to the notification, 1998, of the Government of India, it has been specified that hospital waste management is a part of hospital hygiene and maintenance activities. This involves management of arrange of activities, which are mainly engineering functions such as collection, transportation, operation/treatment of processing systems, and disposal of waste. Initial segregation and storage activities are the direct responsibility of nursing personnel who are engaged in the hospital. ${ }^{[3]}$

More than three-fourth of the health-care wastes are non-hazardous while the remaining proportion is potentially hazardous. According to the WHO, $20 \%$ of total waste generated by healthcare activities are hazardous. ${ }^{[4]}$ 
Dental offices generate a number of hazardous wastes that can be detrimental to the environment if not properly managed. This includes sharps, used disposable items, infectious wastes (blood-soaked cotton, gauze, etc.), mercury-containing waste(mercury and amalgam scrap), lead-containing waste (lead foil packets and lead aprons), and chemical waste (such as spent film developers, fixers, and disinfectants). The success of biomedical waste management (BMW) program depends on the knowledge and practice of the health care worker $(\mathrm{HCW}) .^{[5]}$

With this background, the present study was conducted to evaluate the practical calibration and awareness of dentist and auxiliaries in disposal of hazardous biomedical waste generated during dental treatment into color-coded disposing bags at dental hospital so that depending on their attitude, they can be motivated to attend training and CDE programs concerning waste management that will be efficient to properly segregate, disinfect, and dispose hospital waste in an eco-friendly way.

\section{Aims and Objectives}

The objectives of this were to assess:

1. Awareness toward waste management policy and practices.

2. Approach toward waste management policy and practices.

3. Performance in waste management policy and practices among the private dental practitioners in the city of Vellore, India.

\section{MATERIALS AND METHODS}

\section{Study Design, Area, Duration, and Populations}

The study was a cross-sectional questionnairebased survey which was conducted to identify the exact management problems related to the segregation, collection, transportation, and disposal of hospital waste.

The study design among general dental practitioners and dental specialists was practicing in and around Vellore, Tamil Nadu state, excluding house surgeons, non-practicing dentists, or dentists with administrative job only.

\section{Inclusion Criteria and Exclusion Criteria}

a) Inclusion criteria: Dentists enrolled as a private dental practitioner, willing to participate, and registered in Tamil Nadu state dental directory were included in the study. b) Exclusion criteria: Dental practitioner who was not willing to participate, dental students under internship, and private dental practitioners who were in pilot study were excluded from the study.

\section{Sample Size and Techniques}

A total coverage of dentists working in private clinics in Vellore and surrounding locality were involved. Lists of all private dental clinics were obtained from the directory. The total numbers were 150 registered and practicing dentists.

\section{Survey Tool}

The study was conducted using pre-designed, pre-tested, semi-structured, interview schedule, self-administered, open-ended (27), and close-ended (24) questionnaire with a letter explaining the purpose of the study distributed by the researcher. It was handed to the participants during evening clinic hours. The questionnaire originally developed by Abubakar and Abdo and Abubakar et al. with some modifications. ${ }^{[6,7]}$

In the present investigation, first of all, an extensive pilot study was carried out at 15 dental clinics of the above-mentioned strata which were selected by random sampling technique. The pilot study provided the basic data on which the BMW system was premeditated, designed, and operated in each facility.

Participants were requested to participate voluntary after explanation of the purposes of the study. Informed written consent for their participation was obtained and confidentiality of responses was assured.

\section{Questionnaire}

The questionnaire was formulated into four parts. It consisted of 51 questionnaires with 2responses. They were further categorized into five sections.

Part one

It consisted of six questions of demographic division and qualification data. Dentists were asked about, age, academic qualifications, and years of working in dental clinic or hospital.

Part two

It consisted of seven questions based on the assessment of knowledge on BMW policies and practice. 
Part three BMW

Response consisted of 12 questions based on

Part four

Response consisted of 27 questions based on practices BMW (attitude assessment).

Part five

Response consisted of one question based on preventive measures taken by the health-care professionals while handling biomedical waste.

Participants answered the questionnaire and returned them to the researcher on the same day or the day after. It took 10-15 min to answer all questions.

\section{Data Analysis}

Data were analyzed by SPSS Version 21 (SPSS Inc., Chicago, USA). Results were presented in the form of tables and figures. Comparison between variables assessed using Chi-square and Pearson correlation tests with the level of statistical significance set at $P<0.0001$.

\section{RESULTS}

\section{The Profiles of Respondents [Table 1]}

Descriptive statistics of the results showed that response rate was $100 \%$ (150); the percentage of males was $36 \%(n=54)$ and rest $64 \% \quad(n=96)$ were female. The age group of participants ranged between 25 years and above. Majority of the participants in the present study belonged to the age group of $25-35$ years $(68 \%)(n=102)$. Majority of the participants $(67 \%)(n=101)$ had less than 10 years of practice experience and $83.3 \%(n=125)$ were general dental practitioners.

\section{BMW Policies [Table 2]}

Although majority $(61 \%)(n=92)$ of dentists had heard about guidelines lay down by government of India for BMW, only $50 \%(n=75)$ were aware of Biomedical Waste (Management and Handling) Rules, 1998, and its Amendment Rules were made in 1998.

\section{Response to Knowledge-based Questions on BMW [Table 3]}

About $64.6 \%(n=97)$ of the respondents considered all health-care wastes hazardous. Only $54 \%(n=81)$ were aware of Indian Medical Association Goes Eco-friendly (IMAGE) and of them, only $25 \%(n=38)$ knew the correct expansion of the abbreviation of IMAGE. Around 54\% $(n=81)$ of the respondents correctly recognized the symbol of biohazard. 41\% $(n=62)$ were reported for disinfection of BMW before disposal among them.

\section{Responses to Practice-based Questions on BMW [Table 4]}

The knowledge of waste management guidelines hada significant influence on disposal of dental material $(78 \%)(n=117)$, on disposal of protective

Table 1: Social-demographic variables of respondents

Individual scenario

\begin{tabular}{llcc}
\hline Variables & Respondents & Frequency (n) & Response rate (\%) \\
\hline Total number of respondents & & 150 & 100 \\
Gender & Male & 54 & 36 \\
& Female & 96 & 64 \\
Age group & $25-35$ years & 102 & 68 \\
& $>36$ years & 48 & 32 \\
Years of practice after degree & $<10$ years & 101 & 67.3 \\
& $11-20$ years & 29 & 19.3 \\
Academic qualifications & More than 20 years & 20 & 13.3 \\
Type of practice (job profile) & General practitioner & 125 & 83.3 \\
& Specialist & 25 & 16.6 \\
& Self-employed (private) & 125 & 83.3 \\
& Consultant visiting dental specialist & 16 & 10.6 \\
& Private practitioner (specialist) attached to & 9 & 6
\end{tabular}


Table 2: Biomedical waste management policies

\begin{tabular}{|c|c|c|c|c|c|}
\hline \multicolumn{6}{|c|}{ Individual scenario } \\
\hline Variables & Respondents & $\begin{array}{c}\text { Response } \\
\text { n }(\%)\end{array}$ & Mean \pm SD & Z-value & $\begin{array}{c}\text { Inferential } \\
\text { statistics }\end{array}$ \\
\hline \multirow{3}{*}{$\begin{array}{l}\text { Biomedical Waste (Management and } \\
\text { Handling) Rules were first proposed in }\end{array}$} & 1997 & $31(20.6)$ & \multirow{3}{*}{$50 \pm 22.60$} & \multirow[t]{3}{*}{4.42} & \multirow[t]{3}{*}{$P<0.0001 \mathrm{HS}$} \\
\hline & 1998 & $75(50)$ & & & \\
\hline & 1999 & $44(30)$ & & & \\
\hline \multirow{3}{*}{$\begin{array}{l}\text { Amendments to the Biomedical } \\
\text { Waste (Management and Handling) Rules } \\
\text { were made in }\end{array}$} & 2011 & $50(33.3)$ & \multirow[t]{3}{*}{$50 \pm 7$} & \multirow[t]{3}{*}{14.28} & \multirow[t]{3}{*}{$P<0.0001 \mathrm{HS}$} \\
\hline & 2013 & $43(28.6$ & & & \\
\hline & 2016 & $57(38)$ & & & \\
\hline \multirow{2}{*}{$\begin{array}{l}\text { Biohazard symbol was developed in } 1966 \\
\text { by Charles Baldwin }\end{array}$} & Agree & $62(41.3)$ & \multirow[t]{2}{*}{$75 \pm 18.33$} & \multirow[t]{2}{*}{4.07} & \multirow[t]{2}{*}{$P<0.0001 \mathrm{HS}$} \\
\hline & Disagree & $88(58.6)$ & & & \\
\hline \multirow{2}{*}{$\begin{array}{l}\text { Guidelines lay down by Government of } \\
\text { India for Biomedical Waste Management? }\end{array}$} & Aware & $92(61.3)$ & \multirow[t]{2}{*}{$75 \pm 24.04$} & \multirow[t]{2}{*}{3.11} & \multirow[t]{2}{*}{$P=0.0018 \mathrm{SS}$} \\
\hline & Unaware & $58(38.6$ & & & \\
\hline \multirow{3}{*}{$\begin{array}{l}\text { Regulation of safe transport of medical } \\
\text { waste done by? }\end{array}$} & Pollution Control Board of India & $97(64.4)$ & \multirow[t]{3}{*}{$50 \pm 42.57$} & \multirow[t]{3}{*}{2.34} & \multirow[t]{3}{*}{$P=0.018 \mathrm{SS}$} \\
\hline & Transport Corporation of India & $14(9.3)$ & & & \\
\hline & Cannot comment & $39(26)$ & & & \\
\hline \multirow{3}{*}{$\begin{array}{l}\text { Safe management of biomedical waste is } \\
\text { the responsibility of }\end{array}$} & Only government & $14(9.3)$ & \multirow[t]{3}{*}{$50 \pm 31.95$} & \multirow[t]{3}{*}{3.12} & \multirow[t]{3}{*}{$P=0.0018 \mathrm{SS}$} \\
\hline & Auxiliaries staff & $61(40.6)$ & & & \\
\hline & Dental surgeons & $75(50)$ & & & \\
\hline \multirow{3}{*}{$\begin{array}{l}\text { According to national guidelines, the } \\
\text { maximum time limit for biomedical waste } \\
\text { storage }\end{array}$} & $24 \mathrm{~h}$ & $18(12)$ & \multirow[t]{3}{*}{$50 \pm 38.69$} & \multirow[t]{3}{*}{2.58} & \multirow[t]{3}{*}{$P=0.0098 \mathrm{SS}$} \\
\hline & $48 \mathrm{~h}$ & $39(26)$ & & & \\
\hline & Cannot comment & $93(62)$ & & & \\
\hline
\end{tabular}

NS: Not significant, S: Significant, HS: Highly significant

wears $(40 \%)(n=60)$, and human anatomical wastes $(44 \%)(n=67)$.

It was interesting to note that most injuries (72\%) ( $n=108$ ) occurred during giving injection, which is the most important step of procedure. These were concerned by injuries needle (98\%) $(n=147)$.

With regard to prevention by hepatitis $B$ vaccine, $56.6 \%(n=85)$ of the respondents knew about it and were inoculated.

The most common problem encountered in managing the dental health-care waste was extra expenses, $63.3 \%(n=95)$ of the respondents believed that safe management efforts will increase the financial burden.

\section{Preventive Measures Taken by the Health-Care Professionals While Handling Biomedical Waste [Table 5]}

Use of personal protective measures while handling biomedical waste was adequate among doctors $(46.6 \%)(n=70)$.

\section{DISCUSSION}

The survey presents a grim picture. The study revealed several lacunae in the knowledge, attitudes, and practices among the health professionals. Health professionals have an ethical responsibility toward the environment and themselves. Due to the nature of their profession, they must not forget that they are at risk for treating patients who may have infectious diseases. Dentists, dental assistants, and patients may be exposed to pathogenic microorganisms localized in the oral cavity and respiratory tract, including cytomegalovirus, HBV, HCV, herpes simplex virus Type 1 and 2, HIV, Mycobacterium tuberculosis, Staphylococci, Streptococci, and other viruses and bacteria. ${ }^{[8,9]}$

These microorganisms can be transmitted to dental health-care professionals by direct contact with a patient's saliva, blood, skin, or oral secretions, or by indirect contact through injuries caused by contaminated sharp instruments, or by droplet infection from aerosols or spatter. ${ }^{[10,11]}$ 
Table 3. Response to knowledge-based questions on biomedical waste management

\begin{tabular}{|c|c|c|c|c|c|}
\hline \multicolumn{6}{|c|}{ Individual scenario } \\
\hline Variables & Respondents & $\begin{array}{c}\text { Response } \\
\mathbf{n}(\%)\end{array}$ & Mean \pm SD & Z-value & $\begin{array}{c}\text { Inferential } \\
\text { statistics }\end{array}$ \\
\hline \multirow{2}{*}{$\begin{array}{l}\text { Awareness of biohazard sign/ } \\
\text { IMAGE? }\end{array}$} & Aware & $81(54)$ & $75 \pm 8.48$ & 8.83 & $P<0.0001 \mathrm{HS}$ \\
\hline & Unaware & $69(46)$ & & & \\
\hline Biohazard sign/IMAGE & Designed to warn about & $81(54)$ & $75 \pm 8.48$ & 8.83 & $P<0.0001 \mathrm{HS}$ \\
\hline
\end{tabular}

stands for

Which of the following is the universally accepted symbol for biohazard? hazardous materials

Particular hazard, obstacle, or condition are not covered by a standard sign

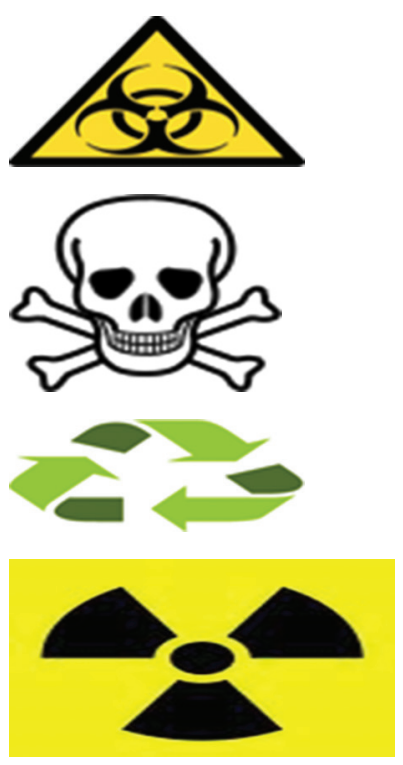

Aware

Awareness of Biomedical Waste Management Rules applicable to dentists?

Awareness of improper waste management causes various health hazards (diseases)?

Biomedical statement describes one type of medical waste?

Biomedical wastes should be segregated into different categories (colored bags)?
Unaware

Aware

Unaware

Materials that may

be poisonous, toxic, or flammable and do not pose disease-related risk

Waste that is saturated to the point of dripping with blood or body fluids contaminated with blood

Waste that does not pose a disease-related risk
Aware
Unaware
$102(68)$

$18(12)$
$22(14.6)$

$37.5 \pm 39.97$

2.81

$P=0.0049$ SS

$30(20)$

$95(63.3)$

$3(2)$

$\begin{array}{llll}65(43.3) & 75 \pm 14.142 & 5.30 & P<0.0001 \mathrm{HS} \\ 85(56.6) & & & \\ 97(64.6) & 75 \pm 31.11 & 2.41 & P=0.0160 \mathrm{SS} \\ 53(35.3) & & & \\ 20(13.3) & 50 \pm 53.70 & 1.86 & P=0.062 \mathrm{NS}\end{array}$

$112(74.6)$

$48(32) \quad 75 \pm 38.18 \quad 1.96 \quad P=0.049$ SS


Table 3. Continued

\begin{tabular}{|c|c|c|c|c|c|}
\hline \multicolumn{6}{|c|}{ Individual scenario } \\
\hline Variables & Respondents & $\begin{array}{c}\text { Response } \\
\text { n }(\%)\end{array}$ & Mean \pm SD & Z-value & $\begin{array}{c}\text { Inferential } \\
\text { statistics }\end{array}$ \\
\hline \multirow{2}{*}{$\begin{array}{l}\text { Infectious waste should be } \\
\text { sterilized from infections by } \\
\text { autoclaving before shredding } \\
\text { and disposal? }\end{array}$} & Agree & $62(41.3)$ & $75 \pm 18.38$ & 4.07 & $P<0.0001 \mathrm{HS}$ \\
\hline & Disagree & $88(58.6)$ & & & \\
\hline \multirow{2}{*}{$\begin{array}{l}\text { Labeling the container before } \\
\text { filling it with waste is of any } \\
\text { clinical significance? }\end{array}$} & Agree & $27(18)$ & $75 \pm 67.88$ & 1.10 & $P=0.026 \mathrm{NS}$ \\
\hline & Disagree & $123(82)$ & & & \\
\hline \multirow{2}{*}{$\begin{array}{l}\text { Can any plastic bag be used } \\
\text { for waste disposal? }\end{array}$} & Agree & $21(14)$ & $75 \pm 76.36$ & 0.98 & $P=0.32 \mathrm{NS}$ \\
\hline & Disagree & $129(86)$ & & & \\
\hline \multirow{2}{*}{$\begin{array}{l}\text { Awareness of amalgam } \\
\text { separators? }\end{array}$} & Aware & $58(38.6)$ & $75 \pm 24.04$ & 3.11 & $P=0.0018 \mathrm{SS}$ \\
\hline & Unaware & $92(61.3)$ & & & \\
\hline \multirow{4}{*}{$\begin{array}{l}1 \mathrm{~g} \text { of mercury (source from } \\
\text { dental amalgam) is enough } \\
\text { to contaminate the following } \\
\text { surface area of a lake? }\end{array}$} & 30 acres & $15(10)$ & $37.5 \pm 38.59$ & 2.91 & $P=0.0036 \mathrm{SS}$ \\
\hline & 25 acres & $92(61.3)$ & & & \\
\hline & 20 acres & $37(24.6)$ & & & \\
\hline & 15 acres & $6(4)$ & & & \\
\hline
\end{tabular}

NS: Not significant, S: Significant, HS: Highly significant, IMAGE: Indian Medical Association Goes Eco-friendly

Self-reported awareness of the BMW system among dentists in the present study (100\%) was good.

Although the self-reported awareness was high, as much as $38.6 \%$ of dentists were not registered at local governing body. Those who had not registered were disposing waste more commonly in dustbins.

Today, hospitals/clinics use a wide variety of drugs including antibiotics, cytotoxics, corrosive chemicals, and radioactive substances, which ultimately become part of hospital waste.

The introduction of disposables in hospitals has brought in its wake many ills such as inappropriate recycling, unauthorized and illegal reuse, and an increase in the quantity of waste. ${ }^{[12]}$

The biomedical waste generated by hospitals and clinics can be broadly categorized as general waste, infectious waste, and non-infectious but hazardous waste. About $75-85 \%$ of waste generated in hospitals is a non-risk or general waste, which constitutes paper, cardboard boxes, plastic packaging, and kitchen waste. Infectious waste, which includes human anatomical wastes, infectious disposable plastic items, and sharps accounts for only remaining $10-15 \%$ of total volume of waste generated in a hospital. Non-infectious but hazardous waste includes chemical waste, genotoxic waste, and radioactive waste which comprises about $5-10 \%$ of total volume of generated hospital waste. ${ }^{[13]}$

Sushma et al. study showed that a substantial percentage of practitioners $(47.9 \%)$ dispose dental waste without segregation and prior disinfection which exposes garbage collectors to a high risk of getting infected from health-care waste which was in accordance with the (64.4\%) present study. ${ }^{[14-16]}$

The Ministry of Environment and Forests, Government of India, has notified the new draft Biomedical Waste (Management and Handling) Rules, 2011, under the Environment Protection Act, 1986, to replace the earlier Biomedical Waste (Management and Handling) Rules, 1998, and amendments thereof. ${ }^{[8]}$ These rules were aware by the health care workers of the present study $(61.3 \%)$.

Regarding the maximum time limit for storage of biomedical waste according to national guidelines, they were not aware of the time limit (62\%) and were aware of the fact that it was $48 \mathrm{~h}$ which was similar to the study by Sood and Sood. ${ }^{[17]}$

IMAGE is the scheme of IMA, Kerala, for the scientific disposal of biomedical waste. IMAGE provides comprehensive service by providing training to hospital staff for segregation of 
Table 4. Response based on practices biomedical waste management

\begin{tabular}{|c|c|c|c|c|c|}
\hline \multicolumn{6}{|c|}{ Individual scenario } \\
\hline Variables & Respondents & Response $n(\%)$ & Mean \pm SD & Z-value & Inferential statistics \\
\hline \multirow{2}{*}{$\begin{array}{l}\text { Does your hospital/clinic } \\
\text { generate biomedical waste? }\end{array}$} & Agree & $150(100)$ & $75 \pm 106.06$ & 0.070 & $P=0.079 \mathrm{NS}$ \\
\hline & Disagree & $0(0)$ & & & \\
\hline \multirow{3}{*}{$\begin{array}{l}\text { Amount of health-care waste } \\
\text { generated per day? }\end{array}$} & $0-2 \mathrm{~kg}$ & $133(88.6)$ & $50 \pm 71.89$ & 1.39 & $P=0.164 \mathrm{NS}$ \\
\hline & $>2-<4 \mathrm{~kg}$ & $10(6.6)$ & & & \\
\hline & $>4 \mathrm{~kg}$ & $7(4.6)$ & & & \\
\hline \multirow{2}{*}{$\begin{array}{l}\text { Does your clinic have a tie-up } \\
\text { with waste management } \\
\text { companies? }\end{array}$} & Agree & $92(61.3)$ & $75 \pm 24.04$ & 3.11 & $P=0.0018 \mathrm{SS}$ \\
\hline & Disagree & $58(38.6)$ & & & \\
\hline \multirow{4}{*}{$\begin{array}{l}\text { Cleaning of dental suction unit } \\
\text { recommended? }\end{array}$} & Daily & $52(34.6)$ & $37.5 \pm 31.05$ & 3.622 & $P<0.0001 \mathrm{HS}$ \\
\hline & Twice a week & $17(11.3)$ & & & \\
\hline & Once a week & $74(49.3)$ & & & \\
\hline & Once a month & $7(4.6)$ & & & \\
\hline \multirow{4}{*}{$\begin{array}{l}\text { Disposal of cotton, gauze, and } \\
\text { other items contaminated by } \\
\text { blood? }\end{array}$} & Red plastic bag & $75(50)$ & $37.5 \pm 30.31$ & 3.711 & $P<0.0001 \mathrm{HS}$ \\
\hline & Yellow plastic bag & $49(32.6)$ & & & \\
\hline & Blue plastic bag & $16(10.6)$ & & & \\
\hline & Black plastic bag & $10(6.6)$ & & & \\
\hline \multirow{4}{*}{$\begin{array}{l}\text { Disposal of pharmaceutical } \\
\text { waste? }\end{array}$} & Red plastic bag & $35(23.3$ & $37.5 \pm 15.54$ & 7.23 & $P<0.0001 \mathrm{HS}$ \\
\hline & Yellow plastic bag & $60(40)$ & & & \\
\hline & Blue plastic bag & $25(16.6$ & & & \\
\hline & Black plastic bag & $30(20)$ & & & \\
\hline \multirow[t]{5}{*}{ Disposal of sharps waste? } & Red plastic bag & $25(16.6)$ & $30 \pm 33.03$ & 3.62 & $P<0.0001 \mathrm{HS}$ \\
\hline & Yellow plastic bag & $12(8)$ & & & \\
\hline & Blue plastic bag & $17(11.3)$ & & & \\
\hline & Black plastic bag & $8(5.3)$ & & & \\
\hline & $\begin{array}{l}\text { Puncture poof } \\
\text { container }\end{array}$ & $88(58.6)$ & & & \\
\hline \multirow{4}{*}{$\begin{array}{l}\text { Disposal of excess mercury } \\
\text { and mercury contaminated } \\
\text { cotton? }\end{array}$} & Drain & $21(14)$ & $37.5 \pm 33.64$ & 3.34 & $P<0.0001 \mathrm{HS}$ \\
\hline & General garbage & $87(58)$ & & & \\
\hline & Plastic bags & $13(8.6)$ & & & \\
\hline & Store in glycerin & $29(19.3)$ & & & \\
\hline \multirow[t]{5}{*}{$\begin{array}{l}\text { Disposal of the used developer } \\
\text { or fixer solution? }\end{array}$} & $\begin{array}{l}\text { Mix and discard } \\
\text { into drain }\end{array}$ & $24(16)$ & $30 \pm 34.35$ & 3.49 & $P<0.0001 \mathrm{HS}$ \\
\hline & $\begin{array}{l}\text { Mix and discard } \\
\text { into general } \\
\text { garbage/plastic } \\
\text { bag }\end{array}$ & $11(7.3)$ & & & \\
\hline & $\begin{array}{l}\text { Discard developer } \\
\text { into drain, send } \\
\text { fixer for recycling }\end{array}$ & $20(13.3)$ & & & \\
\hline & $\begin{array}{l}\text { Discard fixer } \\
\text { into drain, send } \\
\text { developer for } \\
\text { recycling }\end{array}$ & $5(3.3)$ & & & \\
\hline & Cannot comment & $90(60)$ & & & \\
\hline
\end{tabular}




\section{Table 4. Continued}

\begin{tabular}{|c|c|c|c|c|c|}
\hline \multicolumn{6}{|c|}{ Individual scenario } \\
\hline Variables & Respondents & Response $n(\%)$ & Mean \pm SD & Z-value & Inferential statistics \\
\hline \multirow{3}{*}{$\begin{array}{l}\text { Disposal of hazardous liquid } \\
\text { waste? }\end{array}$} & Drain & $36(24)$ & $50 \pm 49.50$ & 2.01 & $P=0.0435 \mathrm{NS}$ \\
\hline & General garbage & $9(6)$ & & & \\
\hline & $\begin{array}{l}\text { Chemical } \\
\text { treatment and } \\
\text { discharge into } \\
\text { drains }\end{array}$ & $105(70)$ & & & \\
\hline \multirow{3}{*}{$\begin{array}{l}\text { Disposal of contaminated } \\
\text { dental materials (files/ } \\
\text { reamers/burs/cements/suction } \\
\text { tips used)? }\end{array}$} & General waste & $15(10)$ & $50 \pm 58.04$ & 1.72 & $P=0.85 \mathrm{NS}$ \\
\hline & Improper manner & $18(12)$ & & & \\
\hline & $\begin{array}{l}\text { Recommended } \\
\text { manner }\end{array}$ & $117(78)$ & & & \\
\hline \multirow{3}{*}{$\begin{array}{l}\text { Disposal of used protective } \\
\text { wears? }\end{array}$} & General waste & $57(38)$ & $50 \pm 14.79$ & 6.76 & $P<0.0001 \mathrm{HS}$ \\
\hline & Improper manner & $33(22)$ & & & \\
\hline & $\begin{array}{l}\text { Recommended } \\
\text { manner }\end{array}$ & $60(40)$ & & & \\
\hline \multirow{3}{*}{$\begin{array}{l}\text { Disposal of human anatomical } \\
\text { waste? }\end{array}$} & General waste & $20(13.3)$ & $50 \pm 26.05$ & 3.83 & \\
\hline & Improper manner & $63(42)$ & & & \\
\hline & $\begin{array}{l}\text { Recommended } \\
\text { manner }\end{array}$ & $67(44.6)$ & & & \\
\hline \multirow{2}{*}{$\begin{array}{l}\text { Disposal of all kinds of waste } \\
\text { into general garbage? }\end{array}$} & Agree & $27(18)$ & $75 \pm 67.88$ & 1.10 & $P=0.26 \mathrm{NS}$ \\
\hline & Disagree & $123(82)$ & & & \\
\hline \multirow{2}{*}{$\begin{array}{l}\text { Is needlestick injury a } \\
\text { concern? }\end{array}$} & Agree & 147 (98) & $75 \pm 101.82$ & 0.73 & $P=0.46 \mathrm{NS}$ \\
\hline & Disagree & $3(2)$ & & & \\
\hline \multirow[t]{3}{*}{ Do you recap the used needle? } & Agree & $142(94.6)$ & $50 \pm 79.69$ & 1.25 & $P=0.209 \mathrm{NS}$ \\
\hline & Disagree & $6(4)$ & & & \\
\hline & Do not bother & $2(1.3)$ & & & \\
\hline \multirow{3}{*}{$\begin{array}{l}\text { Do you discard the used } \\
\text { needle immediately (needle } \\
\text { destroyer)? }\end{array}$} & Agree & $50(33.3)$ & $50 \pm 45$ & 2.22 & $P=0.026 \mathrm{SS}$ \\
\hline & Disagree & $95(63.3)$ & & & \\
\hline & Have not noticed & $5(3.3)$ & & & \\
\hline \multirow{3}{*}{$\begin{array}{l}\text { Sustained a needlestick injury } \\
\text { during the past } 12 \text { months? }\end{array}$} & Agree & $82(54.6)$ & $50 \pm 29.46$ & 3.39 & $P<0.0001 \mathrm{HS}$ \\
\hline & Disagree & $24(16)$ & & & \\
\hline & Do not remember & $44(29.3)$ & & & \\
\hline \multirow{3}{*}{$\begin{array}{l}\text { How the most recent incident } \\
\text { did (sustained a needlestick } \\
\text { injury) happen? }\end{array}$} & $\begin{array}{l}\text { Poor disposal of } \\
\text { needle }\end{array}$ & $22(14.6)$ & $50 \pm 50.23$ & 1.99 & $P=0.046 \mathrm{SS}$ \\
\hline & $\begin{array}{l}\text { Individual } \\
\text { carelessness/ } \\
\text { accident }\end{array}$ & $108(72)$ & & & \\
\hline & Cannot remember & $20(13.3)$ & & & \\
\hline \multirow[t]{2}{*}{$\begin{array}{l}\text { To whom the injury was } \\
\text { reported? }\end{array}$} & $\begin{array}{l}\text { Occupational } \\
\text { health worker }\end{array}$ & $59(39.3)$ & $75 \pm 22.62$ & 3.31 & $P<0.0001 \mathrm{HS}$ \\
\hline & Nobody & $91(60.6)$ & & & \\
\hline \multirow{2}{*}{$\begin{array}{l}\text { Whether fully inoculated } \\
\text { against hepatitis B? }\end{array}$} & Agree & $85(56.6)$ & $75 \pm 14.14$ & 5.30 & $P<0.0001 \mathrm{HS}$ \\
\hline & Disagree & $65(43.3$ & & & \\
\hline
\end{tabular}




\begin{tabular}{|c|c|c|c|c|c|}
\hline \multicolumn{6}{|c|}{ Individual scenario } \\
\hline Variables & Respondents & Response n (\%) & Mean \pm SD & Z-value & Inferential statistics \\
\hline \multirow{2}{*}{$\begin{array}{l}\text { Any previous training } \\
\text { in biomedical waste } \\
\text { management? }\end{array}$} & Agree & $34(22.6)$ & $75 \pm 57.98$ & 1.29 & $P<0.196 \mathrm{NS}$ \\
\hline & Disagree & $116(10.6)$ & & & \\
\hline \multirow{2}{*}{$\begin{array}{l}\text { Biomedical waste management } \\
\text { should compulsorily be made } \\
\text { part of dental undergraduate } \\
\text { curriculum }\end{array}$} & Agree & $141(94)$ & $75 \pm 93.33$ & 0.80 & $P<0.422$ NS \\
\hline & Disagree & $9(6)$ & & & \\
\hline \multirow{2}{*}{$\begin{array}{l}\text { Your knowledge regarding } \\
\text { biomedical waste management } \\
\text { is adequate? }\end{array}$} & Agree & $123(82)$ & $75 \pm 67.88$ & 1.10 & $P=0.26 \mathrm{NS}$ \\
\hline & Disagree & $27(18)$ & & & \\
\hline \multirow{2}{*}{$\begin{array}{l}\text { Any further training } \\
\text { on biomedical waste } \\
\text { management? }\end{array}$} & Required & $145(96.6)$ & $75 \pm 98.99$ & 0.75 & $P=0.44 \mathrm{NS}$ \\
\hline & Not required & $5(3.3)$ & & & \\
\hline \multirow{3}{*}{$\begin{array}{l}\text { Maintaining BMW records } \\
\text { mandatory in your hospital/ } \\
\text { clinic? }\end{array}$} & Agree & $32(21.3)$ & $50 \pm 42$ & 2.38 & $P=0.017 \mathrm{SS}$ \\
\hline & Disagree & $98(65.3)$ & & & \\
\hline & Cannot comment & $20(13.3)$ & & & \\
\hline \multirow{4}{*}{$\begin{array}{l}\text { Problems faced in waste } \\
\text { management? }\end{array}$} & Burden & $35(23.3)$ & $37 \pm 40.57$ & 2.77 & $P=0.0005 \mathrm{SS}$ \\
\hline & Financial burden & $95(63.3)$ & & & \\
\hline & No problem & $3(2)$ & & & \\
\hline & $\begin{array}{l}\text { Non-availability of } \\
\text { service }\end{array}$ & $17(11.3)$ & & & \\
\hline
\end{tabular}

NS: Not significant, S: Significant, HS: Highly significant

Table 5: Preventive measures taken by the health-care professionals while handling biomedical waste

Individual scenario

\begin{tabular}{lcccc}
\hline Preventive measures adopted & Response $\boldsymbol{n}(\boldsymbol{\%})$ & Mean \pm SD & Z-value & Inferential statistics \\
\hline Gloves & $42(28)$ & $30 \pm 28.08$ & 4.27 & HS \\
Goggles & $5(3.3)$ & & \\
Gowns & $31(20) .6$ & & \\
Masks & $2(1.3)$ & & \\
All of the above & $70(46.6)$ & & \\
\hline
\end{tabular}

NS: Not significant, S: Significant, HS: Highly significant

biomedical waste in color-coded bags, collection of it from hospitals, transportation in specially designed covered vehicles, scientific treatment, and final disposal in the common facility. ${ }^{[7]}$ The participants were aware of the IMAGE in the present study.

In a study by Kishore et al. conducted a study in a teaching hospital in New Delhi some 12 years ago, only $35.9 \%$ of respondents were aware of this. However, the guidelines laid down by Government of India for BMW were concerned; it was reassuring to note that only $43.3 \%$ of the dentists were aware of the legislation applicable to hospital waste management. ${ }^{[15,18]}$

More than $68 \%$ of the health care workers had no knowledge of the type of waste to be collected in black-, red-, or yellow-colored bags related to the present study. These findings were similar to the studies done by Sarika et al. which was about $72.5 \%$. ${ }^{[19]}$

Only $14 \%$ of the respondents including the dental students opined that any plastic bag can be used for waste segregation. The observation is in contrast 
with the results of studies done by Charania and Ingle and Sudhir, where the corresponding values were $28 \%$ and $27 \%$, respectively. ${ }^{[11,20]}$

Knowledge of color coding for infectious waste management found poor among BMW staff (32\%). Nasir et al. found the similar result in a study at Faridpur hospital. ${ }^{[21]}$

Sanjeev et al. about $40 \%$ of the respondents were aware of amalgam separators. Amalgam separators are devices designed to remove amalgam waste particles completely in dental office discharge. These separators remove the particles using different techniques such as sedimentation, filtration, centrifugation, or ion exchange which were similar to the present study $(38.6 \%)$. $^{[3]}$

There are no reliable data available of the quantum of waste generated per person per day either in indoors or outdoors patient in Indian Hospital, particularly in Vellore. Even there is no uniformity in the data on the quantum of biomedical waste being generated.

The variation in the quantum of waste generation differs not only from country to country but also within the country which depends on the type of health-care establishment, hospital specialization, proportion of reusable items employed in the healthcare centers, and proportion of patients treated on a day-care basis. ${ }^{[22]}$

From the data available from Zile, where the average daily waste generated of anatomical waste is $5.6 \mathrm{~kg} .{ }^{[22]}$ In the present study, the average daily waste, particularly the anatomical waste, shows high quantity, i.e., $2 \mathrm{~kg} /$ day.

Sudhakar and Chandrashekar conducted among private dental practitioners in Bengaluru city, India, wherein $39.1 \%$ of the respondents were not segregating excess mercury or amalgam but were discarding it to regular garbage. ${ }^{[23]}$ This result is similar to the present study (58\%).

As far as X-ray fixer is concerned, we know the fact that X-ray fixer is considered a hazardous waste due to its high silver content. Developer solutions should not be mixed with fixer solutions. The resulting solution is hazardous. The fixer can be recycled and the developer can be sewered.

In a study by Mushtaq et al., waste X-ray developer and waste X-ray fixer were not collected in separate bottles, rather they are wasted through main wastewater sewage system, therefore, posing health threats it needs revolutionary changes in the ultimate fate of X-ray fixer which was in accordance to the present study $(16 \%) .{ }^{[24]}$

All the surveyed set-ups were found discharging their dangerous waste directly down the drainage waste and also thrown in the garbage. ${ }^{[25-27]}$ These results are in comparison to other studies conducted were similar to the present study.

The BMW management practices in the hospital were satisfactory, except for a deficiency in use of needle cutters in clinics $(63.3 \%)$ which was similar to the study by Mathew et al. (41\%). ${ }^{[28]}$

The practice of reporting of injuries resulting from improperly disposed biomedical waste was found to be completely absent among the staff. Stein et al. in their study reported that among doctors and nurses, only $37 \%$ reported that they ever suffered needlestick injury $39.3 \%$ in the present study. ${ }^{[24]}$

Maroof et al. reported that $43.3 \%$ of the study subjects had heard of hepatitis B. The BWM in the hospital is more in contact with the patients and therefore at greater risk of acquiring Hepatitis B and their knowledge is very deficient which was similar to the present study. ${ }^{[29]}$

However, in the present study, majority of respondents $(22.6 \%)$ had not received any formal training on BMW. Similar result was noted by Akter et al. and Suwarna and Ramesh while performed similar type of research among tertiary HCW. ${ }^{[7,30]}$

The present cross-sectional study was carried out to assess the knowledge and practices of healthcare professionals about BMW in dental clinics. This study showed that of 150 health-care professional's doctors (82\%) had better knowledge compared to other health-care professionals regarding disposal of biomedical waste; this finding was corroborated by the study done by Ramesh et al. (84\%). ${ }^{[31]}$

\section{Recommendations}

a) Adequate supplies and equipment should be available in all departments to take care of wastes.

b) Collected information on various methods of disposal and updated technology should be made available to all categories of health-care personnel.

c) Compulsory training for their health-care personnel from accredited training centers.

d) Easy color coding for BMW disposal bags should be developed in local languages for the 
betterment of sanitary workers and general public awareness.

e) Hospital superintendents, government health administration, and public awareness need to pay their specific attention to this important issue of health and hygiene.

f) Intensive training or workshops program at regular time interval for all staff working in hospitals and clinics, and a system of monitoring and surveillance about practice of day-to-day BMW management should be evolved.

g) Proper BMW disposal practices could be accentuated in health-care personnel if they are put under direct supervision and direct surveillance.

h) Reasonable amount of fund must be provided for waste management.

i) There is dire need of segregation of waste at source besides following color code system of waste management.

j) To install proper incinerators in all the cities

k) Universal precautions should be adapted while dealing with hazardous and infectious waste.

1) Yielding posters with and leaflets should be used to for providing such education.

m) Endorsing the principles of green dentistry which reduces waste and pollution, saves water, energy, and money is high tech and supports a wellness lifestyle.

\section{CONCLUSION}

Occupational safety is a prime concern. Being a recent field of interest, the level of knowledge on this concept is insufficient and needs to be increased to raise awareness of the environmental aspects. Protected and effective execution of waste management rules is not only a legal necessity but also a social liability. Lack of knowledge, motivation, and cost factor are some of the hurdles faced in proper waste management. The importance of training regarding BMW cannot be overemphasized. Health-care professionals and government should work together to develop standard feasible policies for BMW management. The study revealed a lack of knowledge in different tiers of health-care providers which adversely affect their practice.

\section{Ethical Disclosures}

- Protection of human and animal subjects: The author declares that no experiments were performed on humans or animals for this study.

- Confidentiality of data: The author declares that no patient data appear in this article.
- Right to privacy and informed consent: The author has obtained the written informed consent of the patients or subjects mentioned in the article. The corresponding author is in possession of this document.

\section{Conflicts of Interest and Source of Funding}

The author declares that there is no special financial support for this research work from the funding agency and there are no conflicts of interest among all authors.

\section{ACKNOWLEDGMENT}

The author expresses sincere gratitude to all respondents whose honest attention help and support, and the participants of the study lead the research project to worthful outcome.

\section{REFERENCES}

1. Gordon JG, Hardt PA, Denys GA. Medical waste management. In: Mayhall CG, editor. Hospital Epidemiology and Infection Control. $3^{\text {rd }}$ ed. Lippincott Williams and Wilkins Publication; 2004. p. 1773-85.

2. World Health Organization. Safe Management of BioMedical Sharp Waste in India: A Report on Alternative Treatment and Non-Burn Disposal Practices. Geneva: World Health Organization; 2005.

3. Sanjeev R, Suneesh K, Subramaniam R, Prashant PS, Meera G. Knowledge, attitude, and practices about biomedical waste management among dental healthcare personnel in dental college are in Kothamangalam: A crosssectional study. Health Sci 2014;1:1-12.

4. Park K. Hospital waste management. In: Park K, editor. Textbook of Preventive and Social Medicine. Jabalpur, India: Banarasidas Bhanot Publishers; 2009. p. 694-9.

5. Patil GV, Pokhrel K. Biomedical solid waste management in an Indian hospital: A case study. Waste Manag 2005, 25:592-9.

6. Abubakar U, Abdo Y. Hospital waste management in Katsina State and Bayero. J Pure App Sci 2009;2:22-6.

7. Akter N, Kazi NN, Chowdhury AM. Environmental Investigation of Medical Waste Management System in Bangladesh with Reference to Dhaka City. Dhaka: DRAC Research and Evaluation Division. p. 1999. p. 225.

8. Bio-Medical Waste (Management and Handling) Rules; 2011. Available from: http:/www.moef.nic.in/downloads/ publicinformation/salient-features-draft. [Last accessed 2019 Jan 19].

9. Bolyard EA, Tablan OC, Williams WW. Guideline for infection control in health care personnel. Hospital infection control practices advisory committee. Am J Infect Control 1998;26:289-354.

10. Centers for Disease Control and Prevention. Recommended infection-control practices for dentistry. Morb Mortal Wkly Rep 1993;42:1-12. 
11. Charania ZK, Ingle NA. Awareness and practices of dental care waste management among dental practitioners in Chennai city. J Contemp Dent 2011;1:1.

12. Narang RS, Manchanda A, Singh S, Verma N, Padda S. Awareness of biomedical waste management among dental professionals and auxiliary staff in Amritsar, India. Oral Health Dent Manag 2012;11:162-8.

13. Pruss A, Giroult E, Rushbrook P. Safe management of Wastes from Health Care Activities. Geneva: World Health Organization; 1990.

14. Sushma MK, Bhat S, Shetty SR, Babu SG. Biomedical dental waste management and awareness of waste management policy among private dental practitioners in Mangalore city, India. Tanz Dent J 2010;16:39-43.

15. Kishore J, Agarwal R, Kohli C, Sharma PK, Kamat N, Tyagi S, et al. Status of biomedical waste management in nursing homes of New Delhi, India. J Clin Diagn Res 2014;8:56-8.

16. Stein AD, Makarawo TP, Ahmad MF. A survey of doctors and nurses knowledge, attitudes and compliance with infection control guidelines in Birmingham teaching hospitals. J Hosp Infect 2003;54:68-73.

17. Sood AG, Sood A. Dental perspective on biomedical waste and mercury management: A knowledge, attitude, and practice survey. Indian J Dent Res 2011;22:371-5.

18. Kishore J, Goel P, Sagar B, Joshi TK. Awareness about biomedical waste management and infection control among dentists of a teaching hospital in New Delhi, India. Indian J Dent Res 2000;11:157-61.

19. Sarika PP, Murlidhar PT, Prashant JP, Vinod RB. Awareness of healthcare workers regarding biomedical waste management at tertiary care government hospital in Dhule (Maharashtra). Nat J Int Res Med 2013;4:74-9.

20. Sudhir KM. Awareness and practices about dental health care waste management among dentists of Davangere city, Karnataka. J Ind Assoc Pub Health Dent 2006;8:44-50.

21. Nasir UM, Rashedul IM, KhadizaY. Knowledge on hospital waste management among senior staff nurses working in a selected medical college hospital of Bangladesh. J Waste Manag 2014;1:1-5

22. Zile S. Bio-medical waste Management in teaching hospital An experience. J Ind Soc Hosp Waste Manag 2003;2:20-7.

23. Sudhakar V, Chandrashekar J. Dental health care waste disposal among private dental practices in Bangalore city, India. Int Dent J 2008;58:51-4.

24. Mushtaq A, Alam M, Iqbal S, Mushtaq S. Management of dental waste in a dental hospital of Lahore. Biomedica 2008;24:61-3.

25. Hiltz M. The environmental impact of dentistry. J Can Dent Assoc 2007;73:59-62.

26. Ogden GR, Bahrami M, Sivarajasingam V, Phillips G. Dental students knowledge and compliance in cross infection control procedures at a UK dental hospital. Oral Dis 1997;3:25-30.

27. Turnberg WL, Frost F. Survey of occupational exposure of waste industry workers to infectious waste in Washington State. Am J Public Health 1990;80:1262-4.

28. Mathew S, Benjamin AI, Paramita S. Assessment of biomedical waste management practices in a tertiary care teaching hospital in Ludhiana. Health Line 2011;2:5-7.

29. Maroof KA, Bansal R, Parashar P, Sartaj A. Do the medical, dental and nursing students of first year know about hepatits B? A study from a university of North India. J Pak Med Assoc 2012;62:25-7.

30. Suwarna M, Ramesh G. Study about awareness and practices about health care wastes management among hospital staff in a medical college hospital, Bangalore. Int J Basic Med Sci 2012;1:7-11.

31. Ramesh H, Bhagawan BD, Nidhika S. Knowledge and practices regarding biomedical waste management among healthcare professionals in tertiary care hospitals of Mangalore, India. Int $\mathrm{J}$ Com Med Public Health 2015;2:656-9. 